\title{
MANAGEMENT STRATEGIES OF SALINE WATER ON MORPHOMETRIC CHARACTERISTICS OF MELON CULTIVARS
}

\section{JOSÉ F. DE MEDEIROS ${ }^{1}$, CÍCERO P. CORDÃO TERCEIRO NETO ${ }^{2}$, HANS R. GHEYI ${ }^{3}$, NILDO DA S. DIAS ${ }^{4}$, MARIANA S. DE M. SOUZA ${ }^{5}$, RAUNY O. DE SOUZA ${ }^{6}$}

\begin{abstract}
It was to aimed it to investigate effects of various saline water use strategies on melon production and quality of two cultivars (Cucumis melo L., Sancho $-\mathrm{C}_{1}$ and Medellín $-\mathrm{C}_{2}$. The plants were irrigated with water of low $\left(\mathrm{S}_{1}=0.61 \mathrm{dS} \mathrm{m}^{-1}\right)$ and high $\left(\mathrm{S}_{2}=4.78 \mathrm{dS} \mathrm{m}^{-1}\right)$ salinity levels, during each crop stage: $\mathrm{S}_{1} \mathrm{~S}_{1} \mathrm{~S}_{2} \mathrm{~S}_{2}-\mathrm{T}_{1} ; \mathrm{S}_{2} \mathrm{~S}_{1} \mathrm{~S}_{2} \mathrm{~S}_{2}-\mathrm{T}_{2} ; \mathrm{S}_{2} \mathrm{~S}_{2} \mathrm{~S}_{1} \mathrm{~S}_{2}-\mathrm{T}_{3}$. The $1^{\text {st }}, 2^{\text {nd }}, 3^{\text {rd }}$ and $4^{\text {th }}$ terms of these sequences correspond to initial growth, flowering, fruit ripening and harvest phenological stages, respectively. Additionally, there was irrigation rotation during all cycle, with water $S_{1}$ during two days followed by $S_{2}$ for one day $\left(S_{1} 2\right.$ dias $+S_{2} 1$ dia $\left.-T_{4}\right)$ and irrigation with non-salt water $S_{2}$ during all cycle - $T_{5}$. Moreover, we used as control, the irrigation water at $3.2 \mathrm{dS}$ $\mathrm{m}^{-1}$ resulting from water mixture of $S_{1}$ and $S_{2}-T_{6}$ (farm used irrigation management). The experiment was carried out in Pedra Preta Farm, in Mossoró, RN, using an entire randomized block statistical design in a 6x2 subdivided plot scheme with four replications. Saline water irrigation at initial growth stage reduces leaf area and shoot dry phytomass of Sancho and Medellín melon cultivars. The irrigation by $\mathrm{T}_{4}$ provided the highest phytomass production of fruits at 48 DAS, reducing in $33 \%$ of good quality water in irrigation.
\end{abstract}

KEYWORDS: Cucumis melo L., irrigation water quality, growth analysis

\section{ESTRATÉGIAS DE MANEJO DE ÁGUA SALINA SOBRE AS CARACTERÍSTICAS MORFOMÉTRICAS DE CULTIVARES DE MELÃO}

RESUMO: Objetivou-se investigar os efeitos de diferentes estratégias de uso de água salina na produção e na qualidade de frutos de duas cultivares de melão (Cucumis melo L., Sancho - $\mathrm{C}_{1}$ e

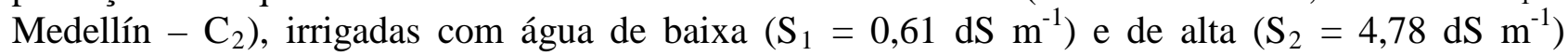
salinidades, por fase da cultura: $S_{1} S_{1} S_{2} S_{2}-T_{1}, S_{2} S_{1} S_{2} S_{2}-T_{2}, S_{2} S_{2} S_{1} S_{2}-T_{3}$ (o $1^{\circ}, 2^{o}, 3^{o}$ e $4^{\circ}$ termos dessas sequências correspondem, respectivamente, às fases fenológicas crescimento inicial, floração, maturação dos frutos e colheita) e a irrigação alternada, em todo o ciclo, com água $\mathrm{S}_{1}$ por 2 dias, seguido por água $S_{2}$ por um dia $\left(S_{1}\right.$ 2dias $+S_{2}$ 1dia $\left.-T_{4}\right)$ e irrigação com água $S_{2}$ em todo o ciclo $-\mathrm{T}_{5}$; além disso, uma testemunha, a irrigação com água de $3,2 \mathrm{dS} \mathrm{m} \mathrm{m}^{-1}$ resultante da mistura das águas $\mathrm{S}_{1}$ e $\mathrm{S}_{2}-\mathrm{T}_{6}$ (manejo adotado pela fazenda ). $\mathrm{O}$ experimento foi conduzido na Fazenda Pedra Preta, no município de Mossoró - RN, utilizando o delineamento em blocos inteiramente casualizados, em um esquema de parcelas subdivididas $6 \times 2$, com quatro repetições. A irrigação com água salobra, logo na fase inicial de crescimento, reduz a área foliar e a fitomassa seca da parte aérea das cultivares de melão Sancho e Medellín. A irrigação do $\mathrm{T}_{4}$ proporcionou a maior produção de fitomassa de frutos de melão aos 48 DAS, reduzindo 33\% de água de boa qualidade na irrigação.

PALAVRAS-CHAVE: Cucumis melo L., Qualidade da água de irrigação, Análise de crescimento.

\footnotetext{
${ }^{1}$ Engenheiro Agrônomo, Prof. Doutor, Departamento de Ciências Ambientais e Tecnológicas Engenharia, UFERSA/Mossoró - RN, Fone: (84) 33151741, jfmedeir@ufersa.edu.br

${ }^{2}$ Engenheiro Agrônomo, Extensionista, EMATER Paraíba, Campina Grande/PB, cicerocordao@yahoo.com.br

${ }^{3}$ Engenheiro Agrônomo, Prof. Visitante Nacional Senior CAPES/UFRB, Núcleo de Engenharia de Água e Solo, UFRB/Cruz das Almas - BA, hans@agriambi.com.br

${ }^{4}$ Engenheiro Agrônomo, Prof. Doutor, Departamento de Ciências Ambientais e Tecnológicas Engenharia, UFERSA/Mossoró - RN, nildo@ufersa.edu.br

${ }^{5}$ Engenheira Agrônoma, Graduada, UFERSA/Mossoró - RN, marianasamira@hotmail.com

${ }^{6}$ Engenheiro Agrônomo, Mestre em Manejo de solo e água, UFERSA/Mossoró - RN, rauny87@hotmail.com

Recebido pelo Conselho Editorial em: 12-2-2012
}

Aprovado pelo Conselho Editorial em: 14-2-2014

Eng. Agríc., Jaboticabal, v.34, n.4, p. 649-659, jul./ago. 2014 


\section{INTRODUCTION}

Rio Grande do Norte State is the second largest Brazilian melon producer, it has been presenting, in recent years, high competitiveness index in domestic and external markets. Amongst factors that favor the vegetable crop, specifically in Mossoró/ Assu, there are soil and weather conditions (soil, temperature and air relative humidity) ideal for crop development.

Mossoró region has as main irrigation water source the groundwater, whose catchment is made through two aquifers. One of them is Açu Sandstone, which presents low salinity water and electrical conductivity $\left(E_{\mathrm{a}}\right)$ that varies between 0.40 and $0.75 \mathrm{dS} \mathrm{m}^{-1}$, the water is catch at $1,000 \mathrm{~m}$ depth what implies in high costs. The other is Jandaíra Limestone, it provides a high salinity level water, whose chemical composition is made of raised concentrations of sodium, calcium, magnesium, bicarbonates and chlorides, and the $\mathrm{EC}_{\mathrm{a}}$ varies from 1.3 to $4.7 \mathrm{dS} \mathrm{m}^{-1}$ and low attainment costs since water is catch at 100-m-depth (PORTO FILHO et al., 2011; SOUSA et al., 2009).

In regional producer opinion, the largest water demand is forcing them to use water at high salinity levels in irrigation since great part of low salinity water is impaired, which is limited to human consumption (GURGEL et al., 2008). However, in order to make this inferior quality water use viable, choosing most salt tolerant and short cycle crops become essential, so harmful effects of salinity would be mitigated (MEDEIROS et al., 2008; AL-KARAKI, 2009; COSME et al., 2011). The main effect that the salinity exerts on plants is divided into three categories: osmotic effects (the high amount of salts in soil solution decrease water availability and then osmotic potential in root zone); specific toxicity of sodium, chloride and boron; and nutritional imbalance (DIAS \& BLANCO, 2010).

Due to genetic variability of melon hybrids, there is a wide variation in salinity tolerance among cultivars. Studies carried out by GURGEL et al. (2010) showed the response to water stress in Goldex cultivar, the authors observed that growth was generally favored with saline water use from 29 to 35 days after sowing, and relative growth rates have been higher. Aragão et al. (2009), evaluating plant tolerance to salinity in three melon cultivars (Sancho, AF 682 and Gaúcho), concluded that salinity negative effect for all evaluated variables was only observed for levels larger than $2 \mathrm{dS} \mathrm{m}^{-1}$ for the three cultivars; however, Sancho was more tolerant compared with AF 682 and Gaúcho.

Porto Filho et al. (2006a) investigated yellow melon (cv. AF 646) under varied salinity levels and observed that shoot absolute growth rate was affected by salinity, whereas shoot relative growth and liquid assimilation rates did not present any effect throughout melon crop cycle.

Besides salinity tolerance differences among species and cultivars of a same species, there might have variations among varieties due to environment adaptation; and still, in a same variety, tolerance level may vary according to phenological stage (DIAS et al., 2011a; DIAS et al., 2011b).

The objective was to determine how different different saline water management, effect vegetative growth of two "Pele de sapo” melon cultivars (Sancho and Medellín).

\section{MATERIAL AND METHODS}

Experiment was carried out during the period of 15 September to 14 November in 2009, it took place in an area of Pedra Preta farm, which belongs to "Coopy Frutas" Agricultural Cooperative Group. The farm lies next to $13 \mathrm{~km}$ of BR 304 highway, which is $28 \mathrm{~km}$ far from Mossoró city. The geographical coordinates are - $4^{\circ} 39^{\prime} 39.24$ ” South latitude; - 37²3' 13.31" West longitude, at a 60-meter altitude. According to Köppen classification adapted to Brazil, the climate is " $B S w h$ " type, which stands for a very hot semiarid tropical climate; it has a rainy station from January summer to behind the fall time in May. Local average temperature is $28.5^{\circ} \mathrm{C}$, with average 
low of $22^{\circ} \mathrm{C}$ and high of $35^{\circ} \mathrm{C}$, annual average rainfall of $673.9 \mathrm{~mm}$ and air relative humidity of $68.9 \%$.

The studied area soil is a Yellow-Red Argisol (EMBRAPA, 2006), a Yellow-Red Ultisols according to U.S. Soil Taxonomy. The soil presents the following physical-chemical attributes: sand = 935.8; silt = 26.5 and clay $=37.7 \mathrm{~g} \mathrm{~kg}^{-1}$ (sandy soil); $\mathrm{pH}_{\mathrm{se}}=7.08 ; \mathrm{P}, \mathrm{Ca}^{2+}, \mathrm{Mg}^{2+}, \mathrm{K}^{+}$and Na contents equal to $5.08 \mathrm{mg} 100 \mathrm{~g}^{-1} ; 2.76 ; 1.29 ; 0.14$ and $0.15 \mathrm{cmol}_{\mathrm{C}} \mathrm{kg}^{-1}$, respectively, which were determined from sample collected at $0-15 \mathrm{~cm}$ of depth through methods recommended by EMBRAPA (1997).

Water used in the research derived from two water-bearing aquifers. They were Açu Sandstone, with depth of approximately $1,000 \mathrm{~m}$, characterized by low salinity water; and Jandaira Limestone, with depth around $80 \mathrm{~m}$ and saline water predominance, having predominance of $\mathrm{Na}^{+}$, $\mathrm{Ca}^{++}$cations and $\mathrm{HCO}_{3}{ }^{-}$and $\mathrm{CL}^{-}$anions. Water chemical analysis is presented in Table 1.

TABLE 1. Chemical characteristics of water applied in the experiment.

\begin{tabular}{|c|c|c|c|c|}
\hline $\begin{array}{c}\text { Chemical } \\
\text { characteristics }\end{array}$ & Units & Water $\mathrm{S}_{1}$ & Water $\mathrm{S}_{2}$ & Water $\mathrm{S}_{0}$ \\
\hline $\mathrm{EC}_{\mathrm{a}}$ & $\mathrm{dS} \mathrm{m}^{-1}$ & 0.61 & 4.78 & 3.21 \\
\hline $\mathrm{pH}$ & - & 6.67 & 6.40 & 6.75 \\
\hline K & $\operatorname{mmol}_{\mathrm{C}} \mathrm{L}^{-1}$ & 0.49 & 0.12 & 0.28 \\
\hline $\mathrm{Na}$ & $\operatorname{mmol}_{\mathrm{c}} \mathrm{L}^{-1}$ & 2.46 & 22.06 & 13.78 \\
\hline $\mathrm{Ca}$ & $\operatorname{mmol}_{\mathrm{c}} \mathrm{L}^{-1}$ & 1.91 & 14.70 & 9.98 \\
\hline $\mathrm{Mg}$ & $\operatorname{mmol}_{\mathrm{C}} \mathrm{L}^{-1}$ & 1.46 & 6.17 & 5.34 \\
\hline $\mathrm{CO}_{3}$ & $\mathrm{mmol}_{\mathrm{c}} \mathrm{L}^{-1}$ & 0.00 & 0.00 & 0.00 \\
\hline $\mathrm{HCO}_{3}$ & $\operatorname{mmol}_{\mathrm{c}} \mathrm{L}^{-1}$ & 3.77 & 4.75 & 4.59 \\
\hline $\mathrm{Cl}$ & $\operatorname{mmol}_{\mathrm{C}} \mathrm{L}^{-1}$ & 1.83 & 38.87 & 23.55 \\
\hline $\mathrm{SO}_{4}$ & Qualitative & $A B S$ & PRES & PRES \\
\hline$\sum$ Cations & $\operatorname{mmol}_{\mathrm{c}} \mathrm{L}^{-1}$ & 6.32 & 43.05 & 29.38 \\
\hline$\sum$ Anions & $\operatorname{mmol}_{\mathrm{c}} \mathrm{L}^{-1}$ & 5.60 & 43.62 & 28.14 \\
\hline SAR* & $\left(\mathrm{mmol} \mathrm{L}^{-11}\right)^{0.5}$ & 1.89 & 6.82 & 4.97 \\
\hline Classification* & & $\mathrm{C}_{2} \mathrm{~S}_{1}$ & $\mathrm{C}_{4} \mathrm{~S}_{1}$ & $\mathrm{C}_{4} \mathrm{~S}_{1}$ \\
\hline
\end{tabular}

* SAR = Sodium Adsorption Ratio. SAR $=\mathrm{Na}^{+} /\left[\left(\mathrm{Ca}^{+2}+\mathrm{Mg}^{+2}\right) / 2\right]^{1 / 2}$

Two melon cultivars, Sancho $\left(\mathrm{C}_{1}\right)$ and Medellín $\left(\mathrm{C}_{2}\right)$, were assessed in trials. The cultivars were irrigated with water of electric conductivity at $0.61\left(\mathrm{~S}_{1}\right)$ and $4.78 \mathrm{dS} \mathrm{m}^{-1}\left(\mathrm{~S}_{2}\right)$ under effect of different management strategies within four crop stages: $S_{1}-S_{1}-S_{2}-S_{2}-T_{1} ; S_{2} S_{1}-S_{2}-S_{2}-T_{2} ; S_{2}-$ $\mathrm{S}_{2}-\mathrm{S}_{1}-\mathrm{S}_{2}-\mathrm{T}_{3}$. The $1^{\text {st }}, 2^{\text {nd }}, 3^{\text {rd }}$ and $4^{\text {th }}$ items of the sequence correspond to growth, flowering, ripening and harvest stages, respectively. Moreover, plants were irrigated by two subsequent water applications $S_{1}$; followed by one successive application $S_{2}$; during all cycle $\left(S_{1}\right.$ 2dias $+S_{2}$ 1dia $\mathrm{T}_{4}$ ); irrigation with $\mathrm{S}_{2}$ water during all cycle $-\mathrm{T}_{5}$; and as control an irrigation with $37 \%$ water $\mathrm{S}_{1}$ and $63 \% \mathrm{~S}_{2}-\mathrm{T}_{6}$, which the last is the water management adopted in the farm.

Growth, flowering, ripening and harvest stages corresponded respectively to the following periods: from transplanting until feminine flower emergence (12 - 30 days after sowing - DAS), up to fruit formation (30 - 46 DAS), unto ripening beginning (46 - 60 DAS) and up to harvest (60 - 75 DAS).

We selected the aforementioned cultivars due to being the most grown and the ones that present great potential to be enlarged. In addition, there is lack of information about techniques on other cultivars, mainly, concerning salinity tolerance. Moreover, these cultivars are well adapted to the regional climatic conditions and have some desirable agronomic characteristics such as disease and pest resistance and post-harvest endurance.

To perform statistical analyses, we used a totally randomized blocks design in a $6 x 2$ subdivided plot scheme with four replications, in a total of 24 plots with $96 \mathrm{~m}^{2}(8.00 \times 12.00 \mathrm{~m})$ and 48 subplots with $48 \mathrm{~m}^{2}(8.00 \times 6.00 \mathrm{~m})$. Each plot was composed of four rows spaced by $2.00 \mathrm{~m}$ 
with $0.50 \mathrm{~m}$ between plants, in a total plant density equivalent to 10,000 plants $\mathrm{ha}^{-1}$. Thus, the evaluated treatments were composed by a combination of saline water irrigation management and cultivar type.

Then, to perform irrigation, we utilized a dripping type system with drips spaced at $0.30 \mathrm{~m}$ and outflow rate of $1.30 \mathrm{~L} \mathrm{~h}^{-1}$. The applied water blade was calculated through estimates from crop evapotranspiration, using Penman-Monteith method that is in accordance with FAO recommendations. Therefore, crop coefficient (Kc) was considered as proposed by FAO, using dual Kc method that adopts as Kcb value: 0.15 at initial, 1.05 at intermediate and 0.75 at final stages.

In relation to soil, the preparation was performed as Pedra Preta farm routine for commercial plantation, consisting of plow, harrow for soil loosening, and subsequent furrow opening and ridging of $1 \times 50 \mathrm{~m}$ for plantation. Fertilization have been made based on soil analyses results, providing an amount of $360 \mathrm{~kg} \mathrm{ha}^{-1}$ of 6-24-12 fertilizer formulation as basal dressing. In the case of covering fertilization, we carried through fertigation since fifth day after transplanting seedlings up to final fruit filling stage with the nutrient amounts of $107 ; 183 ; 235 ; 3.45$ and $2.65 \mathrm{~kg} \mathrm{ha}^{-1}$ of N, $\mathrm{P}_{2} \mathrm{O}_{5}, \mathrm{~K}_{2} \mathrm{O}$, S and $\mathrm{B}$, respectively. As $\mathrm{N}, \mathrm{P}, \mathrm{K}$ sources the fertilizers $\mathrm{KNO}_{3}$, urea, nitric acid, $\mathrm{KCl}$, $\mathrm{K}_{2} \mathrm{SO}_{4}$, MAP, phosphoric acid were used, and as micronutrient sources the boric acid and Quelatec AZ.

Separately, the sowing was carried through in 200 cell trays filled with coconut fiber as substratum (Golden Mix). After 10 days of sowing, we performed seedling transplanting into the field where one seedling was planted per hole. Plant spacing was performed in $2.0 \mathrm{~m}$ between rows and $0.50 \mathrm{~m}$ between plants, at a final density of 10,000 plants ha ${ }^{-1}$.

In order to determine the selected variables of leaf area (LA), shoot dry phytomass (SDP), which was split into branch dry phytomass (BDP) (stem + leaves) and fruit dry phytomass (FDP); we collected four plant samples per subplot at 28, 42, 54 and 69 DAS. To analyze leaf area we used an area integrator LI - COR model LI - 3100 equipment, while for phytomass determination, we dried samples in a forced flow oven at $65^{\circ} \mathrm{C}$ and weighed them in a 0.01 -g precision scale.

Using FA and SDP measurement data obtained throughout the crop cycle, we calculated shoot leaf area rate (SLAR), shoot absolute growth rate (SAGR), shoot relative growth rate (SRGR), and shoot liquid assimilation rate (SLAR). In order to perform the rates calculation, we applied the through the following equations: SFAR $=\mathrm{FA} / \mathrm{SDP}\left(\mathrm{cm}^{2} \mathrm{~g}^{-1}\right)$; SAGR $=\left(\mathrm{SDP}_{1}-\mathrm{SDP}_{2}\right) /\left(\mathrm{T}_{2}-\mathrm{T}_{1}\right)(\mathrm{g}$ day $\left.^{-1}\right) ;$ SRGR = SAGR/SDP $\left(\right.$ gg $^{-1}$ day $\left.^{-1}\right) ;$ SLAR $=$ SRGR/SFAR $\left(\mathrm{g} \mathrm{cm}^{-2}\right.$ day $\left.^{-1}\right)$.

Data were submitted to variance analysis and, when significant, average comparison among treatments was performed by Tukey test at $5 \%$ probability, using the statistical program SISVAR version 4.3 (FERREIRA, 2000).

\section{RESULTS AND DISCUSSION}

According to variance analysis, we observe significant effect of water management factor for the leaf area only at 28 DAS, whereas for the cultivar, there were significant effects for all evaluated periods except at 42 DAS. However, there was no record of significant effect on interaction between management and cultivars (Table 2).

At 28 DAS, melon leaf area on management strategies under low $\mathrm{EC}_{\mathrm{a}}$ irrigation $\left(\mathrm{T}_{1}\right.$ and $\left.\mathrm{T}_{4}\right)$ had no difference among them and control $\left(T_{6}\right)$, being statistically superior to treatments $T_{2}, T_{3}$ and $\mathrm{T}_{5}$. These treatments, in turn, did not present difference, and the smallest leaf areas were reached at them.

There was a leaf area reduction caused by high $\mathrm{EC}_{\mathrm{a}}$ irrigation water at initial growth stage (treatments $T_{2}, T_{3}$ and $T_{5}$ ), what probably have happened by the highest sensitivity to salt of melon plants at this phenological stage. Moreover, it is observed that when water quality is changed from 0.61 to $4.78 \mathrm{dS} \mathrm{m}^{-1}$ since flowering begin until harvest, $\mathrm{T}_{1}$ plant leaf area did not differ from the 
others (Table 2). Then, proving that salinity negative effect on melon besides phenological stage, depend on leaf exposure time, what meet the findings of Dias et al. (2011b).

Further as regards to Table 2, a cultivar analysis has registered leaf area averages of 17,753.65 and 15,102.70 $\mathrm{cm}^{2}$ plant ${ }^{-1}$ at 56 DAS for Sancho and Medellín, respectively. This difference is probably associated to the genetics, once there is no effect of water management and cultivar interaction, in which Medellín achieved the smallest growth in leaf area. Aragão et al. (2009) when assessing salinity tolerance of AF 682, Gaúcho and Sancho cultivars, found that Sancho leaf area reduction had only occurred from $\mathrm{EC}_{\mathrm{a}}$ of $4 \mathrm{dS} \mathrm{m}^{-1}$ on, proving the salt tolerance of this cultivar.

TABLE 2. Variance analysis and average value summary of leaf area at different times for studied treatments.

\begin{tabular}{|c|c|c|c|c|c|}
\hline \multirow{3}{*}{ Variation cause } & \multirow[t]{3}{*}{$\mathrm{DF}$} & \multicolumn{4}{|c|}{ Average square } \\
\hline & & \multicolumn{4}{|c|}{ Days After Sowing (DAS) } \\
\hline & & 28 & 42 & 56 & 69 \\
\hline Water management & 5 & $3369221.92 *$ & $7345641.94^{\mathrm{ns}}$ & $13231701.52^{\mathrm{ns}}$ & $19487056.72^{\mathrm{ns}}$ \\
\hline Block & 3 & $239685.38^{\text {ns }}$ & $20114317.75^{\text {ns }}$ & $26611241.83^{\text {ns }}$ & $27471736.85^{\mathrm{ns}}$ \\
\hline Residue (A) & 15 & 365963.79 & 6682432.45 & 14287516.16 & 13821134.13 \\
\hline Cultivar (C) & 1 & $2134497.83 *$ & $994956.51^{\text {ns }}$ & 84330457.33* & $219947763.12 *$ \\
\hline $\mathrm{M} \times \mathrm{C}$ & 5 & $88425.62^{\mathrm{ns}}$ & $10165529.35^{\mathrm{ns}}$ & $8604615.90^{\mathrm{ns}}$ & 21088517.54 \\
\hline Residue (B) & 18 & 149372.05 & 10108936.08 & 11983247.95 & 20692722.68 \\
\hline VC $1(\%)$ & & 20.34 & 22.58 & 23.01 & 25.08 \\
\hline VC $2(\%)$ & & 13.00 & 27.77 & 21.07 & 30.69 \\
\hline
\end{tabular}

\begin{tabular}{lllll}
\hline Water management & \multicolumn{4}{c}{ Average $\left(\mathrm{cm}^{2}\right)$} \\
\cline { 2 - 5 } $\mathrm{T}_{1}-\mathrm{S}_{1} \mathrm{~S}_{1} \mathrm{~S}_{2} \mathrm{~S}_{2}$ & $3734.90 \mathrm{a}$ & $12025.48 \mathrm{a}$ & $17731.60 \mathrm{a}$ & $16289.81 \mathrm{a}$ \\
$\mathrm{T}_{2}-\mathrm{S}_{2} \mathrm{~S}_{1} \mathrm{~S}_{2} \mathrm{~S}_{2}$ & $2558.91 \mathrm{~b}$ & $11435.57 \mathrm{a}$ & $16884.14 \mathrm{a}$ & $14687.78 \mathrm{a}$ \\
$\mathrm{T}_{3}-\mathrm{S}_{2} \mathrm{~S}_{2} \mathrm{~S}_{1} \mathrm{~S}_{2}$ & $2667.72 \mathrm{~b}$ & $11214.43 \mathrm{a}$ & $14238.55 \mathrm{a}$ & $12894.35 \mathrm{a}$ \\
$\mathrm{T}_{4}-\mathrm{S}_{1} 2 \mathrm{~d}+\mathrm{S}_{2} 1 \mathrm{~d}$ & $3803.35 \mathrm{a}$ & $12966.35 \mathrm{a}$ & $17367.15 \mathrm{a}$ & $16584.85 \mathrm{a}$ \\
$\mathrm{T}_{5}-\mathrm{S}_{2} \mathrm{~S}_{2} \mathrm{~S}_{2} \mathrm{~S}_{2}$ & $2227.00 \mathrm{~b}$ & $10829.61 \mathrm{a}$ & $15645.86 \mathrm{a}$ & $13121.63 \mathrm{a}$ \\
$\mathrm{T}_{6}-\mathrm{S}_{0} \mathrm{~S}_{0} \mathrm{~S}_{0} \mathrm{~S}_{0}$ & $2852.05 \mathrm{ab}$ & $10217.12 \mathrm{a}$ & $16701.74 \mathrm{a}$ & $15362.54 \mathrm{a}$ \\
& \multicolumn{5}{c}{ Average $\left(\mathrm{cm}^{2}\right)$} \\
\hline Cultivars & $3184.86 \mathrm{a}$ & $11592.07 \mathrm{a}$ & $17753.65 \mathrm{a}$ & $16964.11 \mathrm{a}$ \\
$\mathrm{C}_{1}-$ Sancho & $2763.11 \mathrm{~b}$ & $11304.12 \mathrm{a}$ & $15102.70 \mathrm{~b}$ & $12682.87 \mathrm{~b}$ \\
$\mathrm{C}_{2}-$ Medellín & &
\end{tabular}

Same letters in the columns indicate non-significant effect by Tukey test at $5 \%$ of probability; $\mathrm{S}_{1}$ - water from deep well $\left(\mathrm{EC}_{\mathrm{a}}=0.61 \mathrm{dS} \mathrm{m}^{-1}\right) ; \mathrm{S}_{2}$ water from flat well $\left(\mathrm{EC}_{\mathrm{a}}=4.78 \mathrm{dS} \mathrm{m}^{-1}\right) ; \mathrm{S}_{0}-\mathrm{S} 1$ and S2 mixture $\left(\mathrm{EC}_{\mathrm{a}}=3.21 \mathrm{dS} \mathrm{m}^{-1}\right) ;(*)$ : significant at $5 \%$ probability by Tukey test

We noticed non-significant effect on SDP for water management variable in all evaluated periods except for 28 DAS (Table 3), when greater average are observed for low salinity $\left(\mathrm{T}_{1}=\right.$ 24.95 and $\mathrm{T}_{4}=23.72$ g plants $^{-1}$ ), even so differing statistically only from $\mathrm{T}_{5}$ (saline water during all cycle) (Table 4). Comparing cultivar averages, we also observe significant effect at 28, 56 and 69 DAS (Table 3), highlighting Sancho, in which the biggest SDP values were obtained (Table 4). 
TABLE 3. Variance analysis and average value summary of shoot dry phytomass (SDP), branch dry phytomass (BDP) and fruit dry phytomass (FDP) for Sancho and Medellín cultivars at different times.

\begin{tabular}{|c|c|c|c|c|}
\hline \multirow{3}{*}{ Variation causes } & \multicolumn{4}{|c|}{ Average square } \\
\hline & \multicolumn{4}{|c|}{ Days After Sowing (DAS) } \\
\hline & 28 & 42 & 56 & 69 \\
\hline & \multicolumn{4}{|c|}{ ㄴ..1, } \\
\hline Water management $(\mathrm{M})$ & $581.29^{*}$ & $1325.71^{\mathrm{ns}}$ & $10926.24^{\mathrm{ns}}$ & $18407.59^{\mathrm{ns}}$ \\
\hline Block & $311.62^{\mathrm{ns}}$ & $551.47^{\mathrm{ns}}$ & $10369.49^{\mathrm{ns}}$ & $22534.71^{\text {ns }}$ \\
\hline Residue (A) & 1523.17 & 930.35 & 5024.68 & 22128.18 \\
\hline Cultivar (c) & $1151.26^{*}$ & $200.65^{\mathrm{ns}}$ & $40320.45^{*}$ & $117618.93^{\mathrm{ns}}$ \\
\hline $\mathrm{M} \times \mathrm{C}$ & $56.39^{\mathrm{ns}}$ & $1366.85^{\mathrm{ns}}$ & $6952.62^{\mathrm{ns}}$ & $6758.61^{\text {ns }}$ \\
\hline Residue (B) & 189.85 & 1129.51 & 5529.90 & 14193.94 \\
\hline VC $1(\%)$ & 23.38 & 23.21 & 19.88 & 23.45 \\
\hline \multirow[t]{2}{*}{ VC 2 (\%) } & 15.25 & 25.57 & 20.86 & 18.78 \\
\hline & & & .. BDP ...... & \\
\hline Water management (M) & 5 81.29* & $639.53^{\mathrm{ns}}$ & $1754.52^{\mathrm{ns}}$ & $1207.02^{\mathrm{ns}}$ \\
\hline Block & $311.62^{\mathrm{ns}}$ & $991.53^{\mathrm{ns}}$ & $1986.77^{\mathrm{ns}}$ & $1934.05^{\mathrm{ns}}$ \\
\hline Residue (A) & 1523.17 & 537.46 & 1252.59 & 2146.41 \\
\hline Cultivar (c) & 1 151.26* & $263.62^{\mathrm{ns}}$ & 8204.03* & $28561.66^{*}$ \\
\hline $\mathrm{M} \times \mathrm{C}$ & $56.39^{\mathrm{ns}}$ & $637.08^{\mathrm{ns}}$ & $1330.14^{\mathrm{ns}}$ & $1786.54^{\mathrm{ns}}$ \\
\hline Residue (B) & 189.85 & 750.70 & 1395.68 & 1160.57 \\
\hline VC $1(\%)$ & 23.38 & 20.99 & 22.76 & 28.13 \\
\hline \multirow[t]{2}{*}{ VC 2 (\%) } & 15.25 & 24.81 & 24.03 & 20.69 \\
\hline & & & .. FDP. & \\
\hline Water management (M) & $5-$ & $321.57 *$ & $4180.73^{\mathrm{ns}}$ & $16023.29^{\mathrm{ns}}$ \\
\hline Block & $3-$ & $208.16^{\mathrm{ns}}$ & $4349.46^{\text {ns }}$ & $12803.51^{\mathrm{ns}}$ \\
\hline Residue (A) & $15-$ & 96.22 & 3046.97 & 13639.03 \\
\hline Cultivar (c) & $1-$ & $4.29^{\mathrm{ns}}$ & $12149.83^{*}$ & $30261.56^{\text {ns }}$ \\
\hline $\mathrm{M} \times \mathrm{C}$ & $5-$ & $173.38^{\mathrm{ns}}$ & $2686.47^{\mathrm{ns}}$ & $5040.82^{\mathrm{ns}}$ \\
\hline Residue (B) & $18-$ & 128.89 & 2656.84 & 10966.32 \\
\hline VC 1 (\%) & - & 46.71 & 27.46 & 24.86 \\
\hline VC 2 (\%) & - & 54.08 & 25.64 & 22.29 \\
\hline
\end{tabular}

DAS - days after sowing; ns - non-significant at 5\% probability by Tukey test; $\left(^{*}\right)$ - significant at 5\% probability by Tukey test.

Porto Filho et al. (2006b) have observed that there is no significant variation in melon SDP by the saline water use during all crop phenological stages and the continuous use throughout melon cycle. Nevertheless, these authors had used a distinct melon cultivar from the current study, justifying that salt effect on plants might vary among species and cultivars from same species (TRAVASSOS et al., 2012).

With respect to branch dry phytomass accumulation (BDP) (Table 4), it is observed similar behavior to total dry phytomass when evaluating water management. There was a significant effect at 28, 56 and 69 DAS (Table 3) on cultivars similarly to leaf area. In the case of FDP, we evidence a significant effect between cultivars at 56 DAS (Table 3) on fruits, and the highest dry phytomass accumulations represented on average $74 \%$ of the total dry phytomass accumulated (Table 4).

The shoot leafarea rate (SFAR) had significant results in regards of water management only at 28 DAS (Table 5). Hence, when comparing water management treatment averages for SFAR at 28 DAS, we noted that $T_{1}$ was superior to others and statistically equals to $T_{4}$, in turn, have not differed from the other treatments. Briefly, this superiority can be explained by non-application of 
saline water at $T_{1}$ or water switching each two days at $T_{4}$, indicating a certain crop sensitivity at the beginning of growth stage.

In a similar assay, even so with another cultivar, Porto Filho et al. (2006a) also observed a significant effect of salinity on SFAR only at 30 days after sowing. When it comes to water management and cultivars interaction, there was not significant effect at all evaluated times. Yet cultivar factor, it was not observed significant effect at 28, 42 and 56 DAS but significant at 69 DAS, and Sancho cultivar has outpaced Medellín.

TABLE 4. Shoot dry phytomass (SDP), branch dry phytomass (BDP) and fruit dry phytomass (FDP) averages for Sancho and Medellín cultivars at different evaluation periods.

\begin{tabular}{|c|c|c|c|c|}
\hline \multirow{2}{*}{ Water management (M) } & \multicolumn{4}{|c|}{ Days After Sowing (DAS) } \\
\hline & 28 & 42 & 56 & 69 \\
\hline & \multicolumn{4}{|c|}{ SDP (g plant ${ }^{-1}$ ) } \\
\hline $\mathrm{T}_{1}-\mathrm{S}_{1} \mathrm{~S}_{1} \mathrm{~S}_{2} \mathrm{~S}_{2}$ & $23.72 \mathrm{ab}$ & $122.26 \mathrm{a}$ & $404.88 \mathrm{a}$ & $631.62 \mathrm{a}$ \\
\hline $\mathrm{T}_{2}-\mathrm{S}_{2} \mathrm{~S}_{1} \mathrm{~S}_{2} \mathrm{~S}_{2}$ & $18.46 \mathrm{ab}$ & $134.14 \mathrm{a}$ & $342.54 \mathrm{a}$ & $632.67 \mathrm{a}$ \\
\hline $\mathrm{T}_{3}-\mathrm{S}_{2} \mathrm{~S}_{2} \mathrm{~S}_{1} \mathrm{~S}_{2}$ & $19.46 \mathrm{ab}$ & $121.95 \mathrm{a}$ & $304.34 \mathrm{a}$ & $613.45 \mathrm{a}$ \\
\hline $\mathrm{T}_{4}-\mathrm{S}_{1} 2 \mathrm{~d}+\mathrm{S}_{2} 1 \mathrm{~d}$ & 24.95 a & $155.10 \mathrm{a}$ & 349.57 a & $723.85 \mathrm{a}$ \\
\hline $\mathrm{T}_{5}-\mathrm{S}_{2} \mathrm{~S}_{2} \mathrm{~S}_{2} \mathrm{~S}_{2}$ & $16.58 \mathrm{~b}$ & $132.84 \mathrm{a}$ & $344.05 \mathrm{a}$ & $624.90 \mathrm{a}$ \\
\hline $\mathrm{T}_{6}-\mathrm{S}_{0} \mathrm{~S}_{0} \mathrm{~S}_{0} \mathrm{~S}_{0}$ & $20.34 \mathrm{ab}$ & $122.21 \mathrm{a}$ & $393.51 \mathrm{a}$ & 579.95 a \\
\hline \multicolumn{5}{|l|}{ Cultivars } \\
\hline $\mathrm{C}_{1}-$ Sancho & $22.36 \mathrm{a}$ & $133.46 \mathrm{a}$ & $385.46 \mathrm{a}$ & $683.90 \mathrm{a}$ \\
\hline $\mathrm{C}_{2}$ - Medellín & $18.81 \mathrm{~b}$ & 129.37 a & $327.50 \mathrm{~b}$ & $584.91 \mathrm{~b}$ \\
\hline & \multicolumn{4}{|c|}{ BDP $\left(\right.$ g plant $\left.^{-1}\right)$} \\
\hline $\mathrm{T}_{1}-\mathrm{S}_{1} \mathrm{~S}_{1} \mathrm{~S}_{2} \mathrm{~S}_{2}$ & $23.72 \mathrm{ab}$ & $112.10 \mathrm{a}$ & $178.18 \mathrm{a}$ & $173.54 \mathrm{a}$ \\
\hline $\mathrm{T}_{2}-\mathrm{S}_{2} \mathrm{~S}_{1} \mathrm{~S}_{2} \mathrm{~S}_{2}$ & $18.46 \mathrm{ab}$ & $109.16 \mathrm{a}$ & $146.77 \mathrm{a}$ & $173.12 \mathrm{a}$ \\
\hline $\mathrm{T}_{3}-\mathrm{S}_{2} \mathrm{~S}_{2} \mathrm{~S}_{1} \mathrm{~S}_{2}$ & $19.46 \mathrm{ab}$ & $102.40 \mathrm{a}$ & $135.17 \mathrm{a}$ & $147.76 \mathrm{a}$ \\
\hline $\mathrm{T}_{4}-\mathrm{S}_{1} 2 \mathrm{~d}+\mathrm{S}_{2} 1 \mathrm{~d}$ & 24.95 a & $127.06 \mathrm{a}$ & $153.90 \mathrm{a}$ & $174.62 \mathrm{a}$ \\
\hline $\mathrm{T}_{5}-\mathrm{S}_{2} \mathrm{~S}_{2} \mathrm{~S}_{2} \mathrm{~S}_{2}$ & $16.58 \mathrm{~b}$ & $108.45 \mathrm{a}$ & $153.95 \mathrm{a}$ & $150.41 \mathrm{a}$ \\
\hline $\mathrm{T}_{6}-\mathrm{S}_{0} \mathrm{~S}_{0} \mathrm{~S}_{0} \mathrm{~S}_{0}$ & $20.34 \mathrm{ab}$ & 103.34 a & 164.86 a & $168.67 \mathrm{a}$ \\
\hline \multicolumn{5}{|l|}{ Cultivars } \\
\hline $\mathrm{C}_{1}-$ Sancho & $22.36 \mathrm{a}$ & $112.76 \mathrm{a}$ & $168.54 \mathrm{a}$ & $189.08 \mathrm{a}$ \\
\hline \multirow[t]{2}{*}{$\mathrm{C}_{2}$ - Medellín } & $18.81 \mathrm{~b}$ & $108.08 \mathrm{a}$ & $142.40 \mathrm{~b}$ & $140.29 \mathrm{~b}$ \\
\hline & \multicolumn{4}{|c|}{ FDP $\left(\right.$ g plant $^{-1}$ ) } \\
\hline$T_{1}-S_{1} S_{1} S_{2} S_{2}$ & - & $10.16 \mathrm{~b}$ & $226.69 \mathrm{a}$ & $458.08 \mathrm{a}$ \\
\hline $\mathrm{T}_{2}-\mathrm{S}_{2} \mathrm{~S}_{1} \mathrm{~S}_{2} \mathrm{~S}_{2}$ & - & $24.96 \mathrm{ab}$ & $195.76 \mathrm{a}$ & $459.54 \mathrm{a}$ \\
\hline$T_{3}-S_{2} S_{2} S_{1} S_{2}$ & - & $19.55 \mathrm{ab}$ & $169.17 \mathrm{a}$ & $465.68 \mathrm{a}$ \\
\hline $\mathrm{T}_{4}-\mathrm{S}_{1} 2 \mathrm{~d}+\mathrm{S}_{2} 1 \mathrm{~d}$ & - & $28.04 \mathrm{a}$ & 195.67 a & $549.22 \mathrm{a}$ \\
\hline$T_{5}-S_{2} S_{2} S_{2} S_{2}$ & - & $24.38 \mathrm{ab}$ & $190.09 \mathrm{a}$ & $474.49 \mathrm{a}$ \\
\hline $\mathrm{T}_{6}-\mathrm{S}_{0} \mathrm{~S}_{0} \mathrm{~S}_{0} \mathrm{~S}_{0}$ & - & $18.87 \mathrm{ab}$ & 228.65 a & $411.28 \mathrm{a}$ \\
\hline \multicolumn{5}{|l|}{ Cultivars } \\
\hline $\mathrm{C}_{1}$ - Sancho & - & 20.69 a & $216.91 \mathrm{a}$ & $494.82 \mathrm{a}$ \\
\hline $\mathrm{C}_{2}$ - Medellín & - & $21.29 \mathrm{a}$ & $185.10 \mathrm{~b}$ & $444.61 \mathrm{a}$ \\
\hline
\end{tabular}

Same letters in the columns indicate non-significant effect by Tukey test at 5\% of probability; S1 - water from deep well (ECa= 0.61 dS m-1); S2 - water from flat well (ECa= $4.78 \mathrm{dS} \mathrm{m}-1)$; S0 - mixture of S1 and S2 $(\mathrm{ECa}=3.21 \mathrm{dS} \mathrm{m}-1)$. 
TABLE 5 - Shoot leaf area ratio of "Pele de sapo" melon, Sancho and Medellín cultivars, irrigated with saline water under varied management forms.

\begin{tabular}{|c|c|c|c|c|c|}
\hline \multirow{3}{*}{ Variation causes } & \multirow[t]{3}{*}{$\mathrm{DF}$} & \multicolumn{4}{|c|}{ Average square } \\
\hline & & \multicolumn{4}{|c|}{ Days After Sowing (DAS) } \\
\hline & & 28 & 42 & 56 & $* * 69$ \\
\hline$\overline{\text { Water management (M) }}$ & (5) & $767.59^{*}$ & $149.44^{\mathrm{ns}}$ & $86.94^{\mathrm{ns}}$ & $2.37^{\mathrm{ns}}$ \\
\hline Block & (3) & $27.49^{\mathrm{ns}}$ & $143.94^{\mathrm{ns}}$ & $35.22^{\mathrm{ns}}$ & $0.600^{\mathrm{ns}}$ \\
\hline Residue (A) & 15 & 134.57 & 111.44 & 117.27 & 1.016 \\
\hline Cultivar (c) & $(1)$ & $189.07^{\mathrm{ns}}$ & $31.75^{\mathrm{ns}}$ & $23.19^{\mathrm{ns}}$ & $3.810^{*}$ \\
\hline $\mathrm{M} \times \mathrm{C}$ & (5) & $19.02^{\mathrm{ns}}$ & $63.48^{\mathrm{ns}}$ & $55.25^{\mathrm{ns}}$ & $1.031^{\mathrm{ns}}$ \\
\hline Residue (B) & 18 & 103.05 & 254.83 & 93.40 & 0.510 \\
\hline VC 1 (\%) & & 8.04 & 10.16 & 22.97 & 20.25 \\
\hline VC 2 (\%) & & 7.03 & 15.37 & 20.50 & 14.35 \\
\hline \multicolumn{2}{|l|}{$\overline{\text { Water management (M) }}$} & \multicolumn{4}{|c|}{ Average $\left.\left(\mathrm{cm}^{2}\right) \mathrm{g}^{-1}\right)$} \\
\hline $\mathrm{T}_{1}-\mathrm{S}_{1} \mathrm{~S}_{1} \mathrm{~S}_{2} \mathrm{~S}_{2}$ & & $160.08 \mathrm{a}$ & $105.09 \mathrm{a}$ & $44.48 \mathrm{a}$ & $25.23 \mathrm{a}$ \\
\hline$T_{2}-S_{2} S_{1} S_{2} S_{2}$ & & $139.68 \mathrm{~b}$ & $104.91 \mathrm{a}$ & $50.46 \mathrm{a}$ & $36.40 \mathrm{a}$ \\
\hline$T_{3}-S_{2} S_{2} S_{1} S_{2}$ & & $137.39 \mathrm{~b}$ & $111.05 \mathrm{a}$ & $47.91 \mathrm{a}$ & 21.33 a \\
\hline $\mathrm{T}_{4}-\mathrm{S}_{1} 2 \mathrm{~d}+\mathrm{S}_{2} 1 \mathrm{~d}$ & & $152.50 \mathrm{ab}$ & $102.86 \mathrm{a}$ & $50.75 \mathrm{a}$ & 22.78 a \\
\hline $\mathrm{T}_{5}-\mathrm{S}_{2} \mathrm{~S}_{2} \mathrm{~S}_{2} \mathrm{~S}_{2}$ & & $135.13 \mathrm{~b}$ & $100.78 \mathrm{a}$ & $46.81 \mathrm{a}$ & 20.79 a \\
\hline $\mathrm{T}_{6}-\mathrm{S}_{0} \mathrm{~S}_{0} \mathrm{~S}_{0} \mathrm{~S}_{0}$ & & $141.07 \mathrm{~b}$ & $98.51 \mathrm{a}$ & $42.39 \mathrm{a}$ & $28.06 \mathrm{a}$ \\
\hline \multicolumn{2}{|l|}{ Cultivars (C) } & \multicolumn{4}{|c|}{ Average $\left.\left(\mathrm{cm}^{2}\right) \mathrm{g}^{-1}\right)$} \\
\hline $\mathrm{C}_{1}$ - Sancho & & $142.32 \mathrm{a}$ & $103.05 \mathrm{a}$ & $46.44 \mathrm{a}$ & $29.24 \mathrm{a}$ \\
\hline $\mathrm{C}_{2}-$ Medellín & & $146.29 \mathrm{a}$ & $104.68 \mathrm{a}$ & $47.83 \mathrm{a}$ & $22.29 \mathrm{~b}$ \\
\hline
\end{tabular}

Concerning absolute growth rate except for $\mathrm{T}_{3}$ and $\mathrm{T}_{4}$, we verified a growth trend up to the time interval of 42 and 56 DAS, decreasing at the cycle end (Table 6).

Comparing the averages of water handling, at the different crop times, it was noticed significant effect only at the interval between 10 to 28 DAS (Table 7). Treatment $\mathrm{T}_{4}$ presented the largest absolute growth rate (1.37 $\left.\mathrm{g} \mathrm{day}^{-1}\right)$, being statistically equal to $\mathrm{T}_{1}, \mathrm{~T}_{2}, \mathrm{~T}_{3}$, and $\mathrm{T}_{6}$; these in turn, are different from $\mathrm{T}_{5}$, which has presented the lowest rate $\left(0.9 \mathrm{~g} \mathrm{day}^{-1}\right)$, indicating, as well as other variables, greater sensitivity of melon at the growth beginning.

With regard to cultivar type, a significant effect is observed between 10 to 28 and 42 to 56 DAS, and the highest rates of absolute growth belong to Sancho cultivar, even there was no statistical difference in the range of 28 to 42 DAS nor in the last period (56 to 69 DAS) (Table 6). 
TABLE 6. Shoot absolute and relative growth rates (SAGR and SRGR) and shoot liquid assimilation rate (SLAR) for all management types and both cultivars throughout the crop cycle.

\begin{tabular}{|c|c|c|c|c|}
\hline \multirow[t]{2}{*}{$\overline{\text { Water management (M) }}$} & \multicolumn{4}{|c|}{ Days After Sowing (DAS) } \\
\hline & $\overline{10-28}$ & $28-42$ & $42-56$ & $56-69$ \\
\hline & \multicolumn{4}{|c|}{ SAGR $\left(\right.$ g day $\left.^{-1}\right)$} \\
\hline $\mathrm{T}_{1}-\mathrm{S}_{1} \mathrm{~S}_{1} \mathrm{~S}_{2} \mathrm{~S}_{2}$ & $1.31 \mathrm{ab}$ & $6.34 \mathrm{a}$ & $24.36 \mathrm{a}$ & $15.62 \mathrm{a}$ \\
\hline $\mathrm{T}_{2}-\mathrm{S}_{2} \mathrm{~S}_{1} \mathrm{~S}_{2} \mathrm{~S}_{2}$ & $1.01 \mathrm{ab}$ & $6.47 \mathrm{a}$ & $19.44 \mathrm{a}$ & $13.32 \mathrm{a}$ \\
\hline$T_{3}-S_{2} S_{2} S_{1} S_{2}$ & $1.07 \mathrm{ab}$ & $5.92 \mathrm{a}$ & $16.82 \mathrm{a}$ & $20.61 \mathrm{a}$ \\
\hline $\mathrm{T}_{4}-\mathrm{S}_{1} 2 \mathrm{~d}+\mathrm{S}_{2} 1 \mathrm{~d}$ & $1.37 \mathrm{a}$ & $7.29 \mathrm{a}$ & $18.54 \mathrm{a}$ & $24.95 \mathrm{a}$ \\
\hline $\mathrm{T}_{5}-\mathrm{S}_{2} \mathrm{~S}_{2} \mathrm{~S}_{2} \mathrm{~S}_{2}$ & $0.91 \mathrm{~b}$ & $6.56 \mathrm{a}$ & 19.63 a & $18.72 \mathrm{a}$ \\
\hline$T_{6}-S_{0} S_{0} S_{0} S_{0}$ & $1.12 \mathrm{ab}$ & $5.92 \mathrm{a}$ & $24.18 \mathrm{a}$ & $12.64 \mathrm{a}$ \\
\hline \multicolumn{5}{|l|}{ Cultivars (C) } \\
\hline $\mathrm{C}_{1}-$ Sancho & $1.23 \mathrm{a}$ & $6.46 \mathrm{a}$ & $22.71 \mathrm{a}$ & $18.05 \mathrm{a}$ \\
\hline \multirow[t]{2}{*}{$\mathrm{C}_{2}$ - Medellín } & $1.03 \mathrm{~b}$ & $6.37 \mathrm{a}$ & $18.28 \mathrm{~b}$ & $17.23 \mathrm{a}$ \\
\hline & \multicolumn{4}{|c|}{ SRGR $\left(\right.$ g g $^{-1}$ day $\left.^{-1}\right)$} \\
\hline$T_{1}-S_{1} S_{1} S_{2} S_{2}$ & $0.052 \mathrm{a}$ & 0.055 & 0.058 & 0.021 \\
\hline $\mathrm{T}_{2}-\mathrm{S}_{2} \mathrm{~S}_{1} \mathrm{~S}_{2} \mathrm{~S}_{2}$ & $0.055 \mathrm{a}$ & 0.058 & 0.057 & 0.018 \\
\hline $\mathrm{T}_{3}-\mathrm{S}_{2} \mathrm{~S}_{2} \mathrm{~S}_{1} \mathrm{~S}_{2}$ & $0.052 \mathrm{a}$ & 0.056 & 0.055 & 0.031 \\
\hline $\mathrm{T}_{4}-\mathrm{S}_{1} 2 \mathrm{~d}+\mathrm{S}_{2} 1 \mathrm{~d}$ & $0.057 \mathrm{a}$ & 0.057 & 0.050 & 0.035 \\
\hline $\mathrm{T}_{5}-\mathrm{S}_{2} \mathrm{~S}_{2} \mathrm{~S}_{2} \mathrm{~S}_{2}$ & $0.030 \mathrm{~b}$ & 0.060 & 0.053 & 0.027 \\
\hline $\mathrm{T}_{6}-\mathrm{S}_{0} \mathrm{~S}_{0} \mathrm{~S}_{0} \mathrm{~S}_{0}$ & $0.057 \mathrm{a}$ & 0.055 & 0.061 & 0.018 \\
\hline \multicolumn{5}{|l|}{ Cultivars (C) } \\
\hline $\mathrm{C}_{1}-$ Sancho & $0.050 \mathrm{a}$ & $0.056 \mathrm{a}$ & $0.059 \mathrm{a}$ & $0.024 \mathrm{a}$ \\
\hline \multirow[t]{2}{*}{$\mathrm{C}_{2}$ - Medellín } & $0.051 \mathrm{a}$ & $0.057 \mathrm{a}$ & $0.052 \mathrm{~b}$ & $0.026 \mathrm{a}$ \\
\hline & \multicolumn{4}{|c|}{ SLAR $\left(\mathrm{g} \mathrm{m}^{2}\right.$ day $\left.^{-1}\right)$} \\
\hline $\mathrm{T}_{1}-\mathrm{S}_{1} \mathrm{~S}_{1} \mathrm{~S}_{2} \mathrm{~S}_{2}$ & $3.48 \mathrm{c}$ & 5.33 & 13.63 & 9.99 a \\
\hline$T_{2}-S_{2} S_{1} S_{2} S_{2}$ & $3.96 \mathrm{ab}$ & 5.69 & 11.73 & $9.95 \mathrm{a}$ \\
\hline$T_{3}-S_{2} S_{2} S_{1} S_{2}$ & $4.02 \mathrm{ab}$ & 5.32 & 12.24 & $16.90 \mathrm{a}$ \\
\hline $\mathrm{T}_{4}-\mathrm{S}_{1} 2 \mathrm{~d}+\mathrm{S}_{2} 1 \mathrm{~d}$ & 3.63 bc & 5.51 & 10.81 & $15.57 \mathrm{a}$ \\
\hline $\mathrm{T}_{5}-\mathrm{S}_{2} \mathrm{~S}_{2} \mathrm{~S}_{2} \mathrm{~S}_{2}$ & $4.09 \mathrm{a}$ & 6.07 & 12.10 & $14.41 \mathrm{a}$ \\
\hline $\mathrm{T}_{6}-\mathrm{S}_{0} \mathrm{~S}_{0} \mathrm{~S}_{0} \mathrm{~S}_{0}$ & 3.92 abc & 5.75 & 14.58 & $10.97 \mathrm{a}$ \\
\hline \multicolumn{5}{|l|}{ Cultivars (C) } \\
\hline $\mathrm{C}_{1}-$ Sancho & $3.91 \mathrm{a}$ & $5.54 \mathrm{a}$ & $13.07 \mathrm{a}$ & $10.82 \mathrm{a}$ \\
\hline $\mathrm{C}_{2}-$ Medellín & $3.79 \mathrm{a}$ & $5.69 \mathrm{a}$ & $11.96 \mathrm{a}$ & $14.11 \mathrm{a}$ \\
\hline
\end{tabular}

Same letters in the columns indicate non-significant effect by Tukey test at 5\% of probability; S1 - water from deep well (ECa= 0.61 dS m-1); S2 - water from flat well (ECa= $4.78 \mathrm{dS} \mathrm{m}-1)$; S0 - mixture of S1 and S2 (ECa = $3.21 \mathrm{dS} \mathrm{m}-1)$; $(*)$ : significant at $5 \%$ probability by Tukey test

In addition, the growing peak for both cultivars has concentrated in 42 to 56 DAS. Analyzing SRGR, the 10 to 28 DAS interval had significant effect for $T_{1}, T_{2}, T_{3}, T_{4}$ and $T_{6}$, which were similar to each other, differing from $\mathrm{T}_{5}$, where the lowest average was observed $\left(0.030 \mathrm{~g} \mathrm{~g}^{-1} \mathrm{day}^{-1}\right)$ (Table 7). This fact should probably be due to its reduced SAGR rate. Yet, between cultivars, only between 42 and 65 DAS a significant difference was detected, in which Sancho reached the major average $\left(0.059 \mathrm{~g} \mathrm{~g}^{-1} \mathrm{day}^{-1}\right)$. We evidenced no variation for SRGR at the cycle beginning up to 42 to 56 DAS interval, from then on, we observed decreasing rates for all the treatments.

By observing SLAR rate, it became evident that at the crop beginning (10 - 28 DAS), $\mathrm{T}_{5}$ treatment (high salinity) propitiated an enhanced assimilatory gain among treatments $\left(4.09 \mathrm{~g} \mathrm{~m}^{2}\right.$ day $^{-1}$ ), being statistically equal to $T_{2}, T_{3}$ and $T_{6}$ and different of $T_{1}$ and $T_{4}$ (Table 6 ). In other evaluated periods, there was no significant effect for treatments, neither between the cultivars (Table 7). However, along the crop cycle, we noticed greater liquid assimilation rates between 42 
and 56 DAS. After that, $T_{1}, T_{2}$ and $T_{6}$ have decreased. Contrary, for the other treatments $\left(T_{3}, T_{4}\right.$ and $\mathrm{T}_{5}$ ), SLAR had been increased until the last evaluation.

TABLE 7. Variance analysis summary for averages of shoot absolute and relative growth rate (SAGR and SRGR) and shoot liquid assimilation rate (SLAR) of Sancho and Medellín cultivars at the different evaluated periods.

\begin{tabular}{|c|c|c|c|c|c|}
\hline \multirow{3}{*}{ Variation causes } & \multirow[t]{3}{*}{ DF } & \multicolumn{4}{|c|}{ Average square } \\
\hline & & \multicolumn{4}{|c|}{ Days After Sowing (DAS) } \\
\hline & & $10-28$ & $28-42$ & $42-56$ & $56-69$ \\
\hline & & \multicolumn{4}{|c|}{ 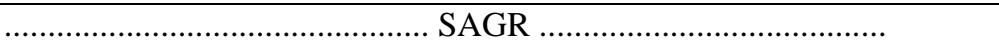 } \\
\hline Water management (M) & (5) & $0.250 *$ & $0.072^{\mathrm{ns}}$ & $0.963^{\mathrm{ns}}$ & $4.93^{\mathrm{ns}}$ \\
\hline Block & (3) & $0.035^{\mathrm{ns}}$ & $0.223^{\mathrm{ns}}$ & $1.283^{\mathrm{ns}}$ & $0.04^{\mathrm{ns}}$ \\
\hline Residue (A) & 15 & 0.070 & 0.090 & 0.458 & 3.01 \\
\hline Cultivar (C) & $(1)$ & $0.472 *$ & $0.0009^{\mathrm{ns}}$ & $3.786 *$ & $0.128^{\mathrm{ns}}$ \\
\hline $\mathrm{M} \times \mathrm{C}$ & (5) & $0.019^{\mathrm{ns}}$ & $0.121^{\mathrm{ns}}$ & $0.484^{\mathrm{ns}}$ & $1.96^{\mathrm{ns}}$ \\
\hline Residue (B) & 18 & 0.030 & 0.158 & 0.693 & 1.93 \\
\hline VC $1(\%)$ & & 23.42 & $* * 11.99$ & $* * 15.23$ & $* * 44.49$ \\
\hline VC $2(\%)$ & & 15.30 & **15.85 & **18.73 & **35.59 \\
\hline Water management (M) & (5) & $0.0043 *$ & $0.00003^{\mathrm{ns}}$ & $0.00012^{\mathrm{ns}}$ & $0.00009^{\mathrm{ns}}$ \\
\hline Block & (3) & $0.00003^{\text {ns }}$ & $0.00004^{\mathrm{ns}}$ & $0.00018^{\mathrm{ns}}$ & $0.00001^{\mathrm{ns}}$ \\
\hline Residue (A) & 15 & 0.0012 & 0.00002 & 0.000062 & 0.00003 \\
\hline Cultivar (C) & $(1)$ & $0.000008^{\mathrm{ns}}$ & $0.00001^{\mathrm{ns}}$ & $0.0006^{*}$ & $0.000008^{\mathrm{ns}}$ \\
\hline $\mathrm{M} \times \mathrm{C}$ & (5) & $0.0002^{\mathrm{ns}}$ & $0.00001^{\mathrm{ns}}$ & $0.00002^{\mathrm{ns}}$ & $0.00003 \mathrm{~ns}$ \\
\hline Residue (B) & 18 & 0.0024 & 0.00003 & 0.00012 & 0.00002 \\
\hline VC $1(\%)$ & & 18.08 & 7.61 & 14.00 & $* * 0.60$ \\
\hline \multirow[t]{2}{*}{ VC 2 (\%) } & & 22.95 & 9.46 & 20.23 & $* * 0.53$ \\
\hline & & \multicolumn{4}{|c|}{. } \\
\hline Water management (M) & (5) & $0.472 *$ & $0.653^{\mathrm{ns}}$ & $0.355^{\text {ns }}$ & $3.812^{\mathrm{ns}}$ \\
\hline Block & (3) & $0.006 \mathrm{~ns}$ & $0.527^{\mathrm{ns}}$ & $0.346^{\mathrm{ns}}$ & $0.212^{\mathrm{ns}}$ \\
\hline Residue (A) & 15 & 0.074 & 0.432 & 0.331 & 1.905 \\
\hline Cultivar (C) & $(1)$ & $0.175 n s$ & $0.296^{\mathrm{ns}}$ & $0.469^{\mathrm{ns}}$ & $3.964^{\mathrm{ns}}$ \\
\hline $\mathrm{M} \times \mathrm{C}$ & $(5)$ & $0.012 \mathrm{~ns}$ & $0.307^{\mathrm{ns}}$ & $0.171^{\mathrm{ns}}$ & $1.760^{\mathrm{ns}}$ \\
\hline Residue (B) & 18 & 0.067 & 0.931 & 0.351 & 1.044 \\
\hline VC 1 (\%) & & 7.07 & 11.70 & $* * 16.48$ & $* * 42.00$ \\
\hline VC 2 (\%) & & 6.74 & 17.18 & $* * 16.98$ & **31.09 \\
\hline
\end{tabular}

DAS - days after sowing; ns - non-significant at 5\% probability by Tukey test; $\left(^{*}\right)$ - significant at $5 \%$ probability by Tukey test; $\left({ }^{* *}\right)$ data transformed into square root of $\mathrm{X}$

\section{CONCLUSIONS}

Firstly, saline water irrigation at initial growth stage reduces leaf area and shoot dry phytomass (leaves and branches) of Sancho and Medellín melon cultivars.

Additionally, non-saline water irrigation applied during 2 days followed by saline water for 1 day provided the highest phytomass production of fruits at 48 DAS, reducing in $33 \%$ the amount of good quality water used in irrigation.

Finally, saline water use in melon crop irrigation during all the cycle reduces the absolute growth rate for Sancho and Medellín cultivars, being the initial growing stage the most sensible to this saline water.

\section{REFERENCES}

AL-KARAKI, G.; AL-AJMI, A.; OTHMAN, Y. Response of soilless grown bell pepper cultivars to salinity. Acta Horticulturae, The Hague, v.807, p.227-232, 2009.

ARAGÃO, C. A.; SANTOS, J. S.; QUEIROZ, S. O. P; FRANÇA, B. Avaliação de cultivares de melão sob condições de estresse salino. Revista Caatinga, Mossoró, v.22, n.2, p.161-169, 2009. 
COSME, C.R.; DIAS, N.S.; OLIVEIRA, A.M.; OLIVEIRA, E.M.M.; SOUSA NETO, O.N. Produção de tomate hidropônico utilizando rejeito da dessalinização na solução nutritiva aplicados em diferentes épocas. Revista Brasileira de Engenharia Agrícola e Ambiental, Campina Grande, v.15, n.5, p.499-504, 2011.

DIAS, N.S.; LIMA, F.A.; SILVA, C.R.; SOUSA NETO, O.N.; GHEYI, H.R.. Use of reject brine from desalination on different development stages of hydroponic lettuce. Revista Caatinga, Mossoró, v.24, n.1, p.76-81, 2011a.

DIAS, N.S.; OLIVEIRA, A.M.; SOUSA NETO, O.N.; BLANCO, F.F.; REBOUCAS, J.R.L. Concentração salina e fases de exposição à salinidade do meloeiro cultivado em substrato de fibra de coco. Revista Brasileira de Fruticultura, Jaboticabal, v.33, n.4, p. 915-921, 2011 b.

DIAS, N. S.; BLANCO, F. F. Efeitos dos sais no solo e na planta. In: GHEYI, H. R.; DIAS, N. da S.; LACERDA, C. F. da (Org.). Manejo da salinidade na agricultura: estudos básicos e aplicados. Fortaleza: INCTSal, 2010. v.1, p.129-141.

EMBRAPA. Centro Nacional de Pesquisa de Solos. Manual de métodos de análise de solo. 2.ed. Rio de Janeiro, 1997. 212p. (Documentos, 1).

EMBRAPA. Sistema brasileiro de classificação de solos. 2.ed. Rio de Janeiro, 2006. 306p.

FERREIRA, D. F. Manual do sistema SISVAR para análises estatísticas. Lavras: UFLA, 2000. 66p.

GURGEL, M. T., UYEDA, C. A., GHEYI, H. R., OLIVEIRA, F. H. T., FERNANDES, P. D.; SILVA, F. V. Crescimento de meloeiro sob estresse salino e doses de potássio. Revista Brasileira de Engenharia Agrícola e Ambiental, Campina Grande, v.14, n.1, p.3-10, 2010.

GURGEL, M. T.; GHEYI, H. R.; OLIVEIRA, F. H. T.; FERNANDES, P. D.; SILVA, F. V. Nutrição de cultivares de meloeiro irrigadas com águas de baixa e alta salinidade. Revista Caatinga, Mossoró, v.21, n.1, p.36-43, 2008.

MEDEIROS, J. F; DUARTE, S. R; FERNANDES, P. D; DIAS, N. S; GHEYI, H. R. Crescimento e acúmulo de N, P e K pelo meloeiro irrigado com água salina. Horticultura Brasileira, Brasilia, v.26, n.3, p.452-457, 2008.

PORTO FILHO, F. Q.; MEDEIROS, J. F.; GHEYI, H.R.; MATOS, J. A.; SOUZA, E. R.; SOUSA NETO, E. R. Crescimento do meloeiro irrigado com águas de diferentes salinidades. Horticultura Brasileira, Brasilia, v.24, n.3, p.334-341. 2006a.

PORTO FILHO, F. Q.; MEDEIROS, J. F.; SOUSA NETO, E. R.; GHEYI, H. R.; MATOS J. A. Viabilidade da irrigação do meloeiro com águas salinas em diferentes fases fenológicas. Ciência Rural, Santa Maria, v.36, n.2, p.453-459, $2006 \mathrm{~b}$.

PORTO FILHO, F. Q.; MEDEIROS, J. F.; GHEYI, H. R.; DIAS, N. S.; SOUSA, P. S.; DANTAS, D. C. Evolução da salinidade e do $\mathrm{pH}$ de um solo sob cultivo de melão irrigado com água salina. Revista Brasileira de Engenharia Agrícola e Ambiental, Campina Grande, v.15, n.11, p.1130-1137, 2011.

SOUSA, R. B. C.; OLIVEIRA, L. M.; CARVALHO, J. W. C.; DIAS, N. S.; GURGEL, M. T. Variação sazonal das águas subterrâneas utilizadas para irrigação na microrregião de Tibau, RN. Revista Caatinga, Mossoró, v.22, n.4, p.206-213, 2009.

TRAVASSOS, K. D.; SOARES, F.A.L.; BARROS, H. M. M. ; DIAS, N. S.; UYEDA, C. A.; SILVA, F. V. Crescimento e desenvolvimento de variedades de girassol irrigado com água salina. Irriga, Botucatu, v.1, p.324-339, 2012.Edição Especial. 